\title{
SELECCIÓN DE CULTIVARES FORRAJEROS DE SORGO (Sorghum bicolor) Y MIJO (Pennisetum americanum) POR ÍNDICES DE EFICIENCIA DE PRODUCCIÓN Y CALIDAD 1
}

\author{
Jaime Ruiz Vega' ${ }^{2}$ José Cruz Carrillo Rodríguez ${ }^{3}$
}

\begin{abstract}
RESUMEN
Selección de cultivares forrajeros de sorgo (Sorghum bicolor) y mijo (Pennisetum americanum) por índices de eficiencia de producción y calidad. Con el propósito de identificar cultivares de sorgo y mijo forrajeros con alta eficiencia de producción de materia seca y calidad para condiciones de riego restringido, situación cada vez mas frecuente debido al abatimiento de los niveles freáticos en el período primaveraverano, se sembraron el 23 de marzo de 1998 en Santa Cruz Xoxocotlán, Oaxaca, México, seis variedades comerciales de sorgo forrajero (Sorghum bicolor) y una de mijo perla (Pennisetum americanum), además de seis líneas de mijo perla provenientes del International Crop Research Institute for the Semiarid Tropics (ICRISAT), Hyderabad, India. A partir de los datos de producción de materia seca, se generaron dos índices, uno llamado de productividad modificado (IPM) y otro de productividad (IP), para facilitar la selección de materiales. El IPM superó al IP en su capacidad para discriminar los materiales por mayor eficiencia de producción de forraje de calidad. En promedio, los genotipos de sorgo fueron más eficientes para producir materia seca, especialmente Sweet Sioux y Domor, este último caracterizado por mostrar poca variación entre cortes. Sin embargo, los mijos NELC C4 y ICMH 423, podrían ser utilizados por campesinos que dispongan de menos agua y tengan urgencia de forraje, dada su alta eficiencia de producción de materia seca de buena calidad en el primer corte.
\end{abstract}

Palabras clave: Fenología del cultivo, unidades de calor, índices de selección, sorgo, mijo.

\begin{abstract}
Selection of sorghum (Sorghum bicolor) and millet (Pennisetum americanum) cultivars by production efficiency and forage quality indexes. Six commercial varieties of sorghum (Sorghum bicolor), one of pearl millet (Pennisetum americanum), and six millet lines from The International Crop Research Institute for the Semiarid Tropics (ICRISAT), Hyderabad, India, were planted on March 23, 1998 in Santa Cruz Xoxocotlán, Oax., México, to identify materials of sorghum and millet with higher production efficiency and forage quality under conditions of restricted irrigation, as a result of generalized lower water table levels during the spring-summer period. To facilitate the selection of varieties, productivity and modified quality indexes were generated from dry matter production data. The IPM was more adequate than the IP to discriminate the varieties for their forage production efficiency and quality. On average, the sorghum genotypes, especially Sweet Sioux and Domor, the last one showing little variation between harvests, were more efficient in dry matter production than the millet ones. However, because of their high production efficiency and quality at the first harvest, the millet cultivars NELC C4 and ICMH 423 could be recommended for farmers which need forage urgently and have less water availability.
\end{abstract}

Key words: Crop phenology, heat units, selection indexes, sorghum, millet.

\footnotetext{
1 Recibido: 24 de febrero, 2005. Aceptado: 30 de agosto , 2005.

2 Profesor Investigador del CIIDIR OAXACA, Calle Hornos 1003, Sta. Cruz Xoxocotlán, Oax. CP 71230. México. Correo electrónico: jvega@ipn.mx, jruizv@yahoo.com

3 Profesor de Maestría, ITAO, Nazareno Xoxocotlán, Oax. Apdo. Postal 263, CP 68000. México. Correo electrónico: carrijos@ prodigy.net.mx
} 


\section{INTRODUCCIÓN}

En la región de los valles centrales de Oaxaca existe una producción de forraje cercana a las $125.000 \mathrm{t}$ de materia seca por año, incluyendo principalmente alfalfa (Medicago sativa), maíz forrajero (Zea mays), sorgo y rastrojos de maíz y frijol (Phaseolus vulgaris) (SAGDR 1995). Sin embargo, debido al incremento de los hatos ganaderos, la incidencia frecuente de sequías y la escasez de agua para riego, se estima un déficit de más del $70 \%$ en el período invierno-verano (López et al. 1992).

La cantidad de materia seca producida por una planta es dependiente de una gama de factores ambientales y genéticos. Dentro de los ambientales, se incluye la luz, $\mathrm{CO}_{2}$, temperatura, humedad disponible y nutrientes; mientras que, en los genéticos se incluye al tipo de fotosíntesis, la estructura del dosel y el índice de área foliar (Hopkins 1999). La mayor parte de las especies de mijo y sorgo son plantas de fotosíntesis C4. Sin embargo, la deficiencia de humedad es uno de los principales factores que a nivel mundial limitan los rendimientos, aún en zonas templado-húmedas (Raper y Kramer 1983).

Los mijos son muy resistentes a sequía y a suelos pobres (Lowenberg-DeBoer et al. 1995; Tapia 1985), debido a mecanismos de escape o de tolerancia El sorgo destaca también por su alta tolerancia a la sequía, especialmente en la etapa vegetativa, pues puede recuperarse de sequías impuestas incluso en la etapa de diferenciación floral sin menoscabos en el rendimiento (Castro et al. 2000). Aún así, se pueden observar disminuciones en las tasas fotosintéticas durante el mediodía, debido a bajos potenciales hídricos asociados a altas tasas transpiratorias (Hirasawa y Hsiao 1999), pero éstas no son tan acusadas como en las plantas C3, debido a la presencia de estructuras vasculares que conforman la anatomía tipo "Kranz" (Gliessman 2000).

Sin embargo, la anatomía tipo "Kranz", presente en las plantas con fotosíntesis tipo $\mathrm{C} 4$, se asocia con una menor calidad del forraje debido a la presencia de estructuras lignificadas y menores volúmenes de mesofilo (Wilson et al. 1983), pero para géneros donde existan especies con características entre $\mathrm{C} 3$ y C4, la digestibilidad de las especies C4 podría mejorarse por hibridación (Bohn et al. 1988).

Los mijos y sorgos forrajeros pueden producir de 18-25 t/ha de forraje seco, por corte. Sin embargo, la calidad del forraje es mayor en los mijos, y no presentan problemas de producción de ácido prúsico, sustancia tóxica para el ganado (CIAN 1984; Báez 1988). El porcentaje de materia seca digestible in vitro (MSDIV) en los mijos puede alcanzar el $69 \%$, mientras que en los sorgos forrajeros es de cerca del $66 \%$ (Banks y Stewart 1998). Los sorgos con nervadura café tienen valores cercanos al $80 \%$, comparable con valores para maíz forrajero (McCollum y Bean 2003). Sin embargo, en Torreón, Coahuila, México, se encontró que aunque el sorgo con nervadura café tenía mayor porcentaje de materia seca digestible que el sorgo normal, los rendimientos totales por hectárea de MSDIV eran mayores en este último (Nuñez y Cantú-Brito 2000). El porcentaje de proteína cruda en sorgo se ha estimado en 5,2, contra 9,9 del mijo (Muir et al. 1999). También el mijo se reconoce como más resistente a sequía, al menos en la etapa vegetativa (Hopkins 1999; Beets 1985).

La calidad del forraje está determinada por el contenido de nutrientes y su digestibilidad (Cherney y Martin 1980). Algunos indicadores indirectos son el porcentaje de proteína digestible, fibra cruda, nutrientes asimilables totales y contenido de minerales. Sin embargo, la mejor evaluación de la calidad de los forrajes se obtiene a través de su efecto sobre la productividad del ganado, incluyendo la producción de leche, ganancia de peso y eficiencia reproductiva (Pinkerton et al. 1991).

El porcentaje de hojas se relaciona directamente con la calidad del forraje, ya que el valor nutritivo de éstas es mayor que el de los tallos (White et al. 1981). Sin embargo, la sequía (Castro et al. 2000; Bittma et al. 1988) y otros factores (Chapman y Lemaire 1993) puede acelerar el envejecimiento de las hojas, haciéndolas menos digestibles. El contenido de sustancias albuminoides es mayor en las plantas jóvenes; mientras que, el contenido de fibra se incrementa con la edad, por lo que se considera que la mejor calidad se obtiene cortando el forraje al 50 \% de floración (Robles 1983; Báez 1988).

La producción total de materia seca no es un buen criterio para la selección de materiales forrajeros, esta debería hacerse con base en parámetros indicadores tanto de la productividad (Stoskopfv 1981; Ruiz 1997) como de la calidad del forraje (Báez 1988). En muchas plantas forrajeras, una relación alta de peso de tallos/ hojas resulta en una menor digestibilidad (Fales 1986). En zonas semiáridas también es importante considerar la eficiencia de producción por unidad de agua disponible (Fischer 1980).

El objetivo del presente trabajo fue el de determinar índices para la selección más eficiente de genotipos forrajeros bajo condiciones de riego de auxilio en zonas con disponibilidad restringida de agua, donde generalmente los riegos se aplican hasta que el cultivo muestra síntomas de sequía. 


\section{MATERIALES Y MÉTODOS}

El experimento se estableció en Nazareno Xoxocotlán, Oaxaca $\left(16^{\circ} 06^{\prime} \mathrm{N}, 95^{\circ} 54^{\prime} \mathrm{O}, 1.575 \mathrm{~m}\right.$ de altitud, temperatura y precipitación medias anuales de $21,8^{\circ} \mathrm{C}$ y $669 \mathrm{~mm}$ ), el 23 de marzo de 1998, en un suelo profundo de origen fluvial, de textura arcillo-limosa, bajo en materia orgánica, con un $\mathrm{pH}$ ligeramente alcalino y de fertilidad moderada.

Se utilizaron seis variedades comerciales de sorgo forrajero (Sweet Sioux, Beefbuilder-T, Grazer, Cow Vittles, Dine a Mite y Domor); una de mijo perla (Pabellón 88 ), además de seis líneas de mijo perla (NELC C4, ICMH 83401, ICMH 423, ICMS 8283, ICMS 7704 e ICTP 8203) provenientes del International Crop Research Institute for the Semiarid Tropics (ICRISAT), Hyderabad, India. La densidad de siembra para los genotipos de sorgo fue de $15 \mathrm{~kg} / \mathrm{ha}$, mientras que para los mijos fue de $25 \mathrm{~kg} / \mathrm{ha}$, lo cual correspondió a densidades medias de población de 834.000 y 893.000 plantas/ha, respectivamente.

Al momento de la siembra se aplicó la fórmula de fertilización 85-60-00 (kg/ha de $\left.\mathrm{N}-\mathrm{P}_{2} \mathrm{O}_{5}-\mathrm{K}_{2} \mathrm{O}\right)$, para posteriormente aplicar otros $85 \mathrm{~kg} / \mathrm{ha}$ de nitrógeno al momento de la primera labor, 20 días después. Como fuente de nitrógeno se utilizó urea y para fósforo se utilizó superfosfato de calcio triple. Se aplicaron un riego de siembra y cuatro riegos de auxilio, cada uno de 15 $\mathrm{cm}$ aproximadamente, del 27 de marzo al 2 de junio, ya que durante este período no se presentan precipitaciones significativas en la región. Los riegos se aplicaron cuando el cultivo mostraba síntomas de marchitez moderada durante el mediodía, práctica común entre los agricultores del área.

Se empleó un diseño experimental en bloques al azar con cuatro repeticiones y parcelas experimentales útiles de tres surcos de $3 \mathrm{~m}$ de largo y $70 \mathrm{~cm}$ de ancho. La parcela experimental constó de cinco surcos de $5 \mathrm{~m}$ de largo.

Durante el desarrollo del experimento se registraron las siguientes variables:

Días a emergencia: definido como el momento en el cual emergen el $50 \%$ de las plántulas.

Días a tres hojas: cuando el $50 \%$ de las plantas tenían tres hojas liguladas visibles.

Días al encañe: cuando se observó el primer nudo visible en $50 \%$ de las plantas.

Días al embuche: ocurre cuando en el $50 \%$ de las plantas la panícula presenta un crecimiento de $3 \mathrm{~cm}$, para evaluarlos fue necesario abrir la parte superior de las plantas.

Días al $50 \%$ de floración: cuando el $50 \%$ de las plantas tenían inflorescencia visible.

Rendimiento de forraje seco al $50 \%$ de floración (RFS): Se cosechó la parcela útil, consistente en tres surcos centrales de $3 \mathrm{~m}$ de longitud para la medición de la producción de forraje verde. Una muestra de éste se secó hasta peso constante en una estufa a $70{ }^{\circ} \mathrm{C}$ para estimar el porcentaje de materia seca presente. Se realizaron dos cortes, el primero se efectuó 54 a 70 días después de la siembra, mientras que el segundo se realizó de 24 a 58 días después del primero, dependiendo de la precocidad de los genotipos.

Área foliar al 50 \% de floración: Se midió a partir de la lámina foliar de todas las plantas presentes en 0,50 m de surco al momento del muestreo, utilizando la relación (Stickler et al. 1961):

$$
A=\operatorname{largo} x \text { ancho de hojas } x 0,75
$$

Temperaturas máximas y mínimas: Medidas diariamente con un termómetro tipo "six" (Brannan, England) al abrigo.

A partir de dichas variables se generaron las siguientes:

Eficiencia de Producción de Materia Seca (EPMS): se estimó dividiendo la producción de materia seca sobre los días al corte.

Indice de Área Foliar o área de hojas por unidad de superficie $=$ IAF .

Índice de Cosecha (IC) o \% de hojas: equivale al peso seco del follaje sobre el peso seco total de la parte aérea; un indicador indirecto de la calidad del forraje (Fales 1986).

\section{Indice de Productividad (IP):}

IP = rendimiento biológico o RFS + índice de Cosecha, una adecuación del índice propuesto por Stoskopfv (1981), ya que no se midió rendimiento de grano.

\section{Indice de Productividad Modificado (IPM):}

$\mathrm{IPM}=\mathrm{EPMS}+$ Indice de cosecha

Unidades de calor (UC): Obtenidas por el método residual, utilizando una temperatura base de $15^{\circ} \mathrm{C}$, es decir,

AGRONOMÍA MESOAMERICANA 16(2): 153-160. 2005 


$$
U C=\Sigma\{(\text { Tmax }- \text { Tmin }) / 2\}-15,0
$$

El análisis estadístico se realizó utilizando el paquete "Statistical Analysis System", SAS (1994), incluyó análisis de varianza, comparaciones múltiples de medias (Tukey al 0,05 de probabilidad), así como análisis de regresión y correlación.

\section{RESULTADOS Y DISCUSIÓN}

\section{Fenología y producción de materia seca}

Debido a la pertinencia de la información para este artículo, a continuación se citan los resultados obtenidos para estas variables en un artículo previo (Carrillo y Ruiz 2004). En cuanto a los días a emergencia, días a tres hojas y días al embuche, los mijos resultaron más precoces que el grupo de genotipos de sorgo. El sorgo más tardío fue Sweet Sioux con 58,25 días al embuche, mientras que el más precoz fue Domor con 48,5 días. El cultivar NELC C4, con 48,5 días al embuche, fue el más tardío de los mijos, siendo el más precoz $\mathrm{Pa}$ bellón 88 con 38,7 días.

Por lo que respecta a producción de materia seca, en el primer corte, realizado cuando las plantas iniciaron floración, NELC C4 produjo la misma cantidad de materia seca que el genotipo de sorgo Beefbuilder-T, pero en el segundo corte su rendimiento disminuyó drásticamente, al igual que el de los otros mijos . Considerando los dos cortes, los sorgos forrajeros más sobresalientes fueron Sweet Sioux y Beefbuilder-T, con una producción total de alrededor de 32 y 30 t/ha de materia seca, respectivamente. Estos fueron los materiales mas tardíos.

\section{Días al corte}

El número de días al $50 \%$ de floración o días al corte fue mayor en el grupo de las variedades de sorgo. Sin embargo, en la práctica, los días al primer corte solo fueron diferentes entre el sorgo más tardío y el mijo más precoz, ya que para el resto de materiales el rango fue de 59-63 días (Cuadro 1). Para el segundo corte, los días a $50 \%$ de floración disminuyeron, especialmente en los mijos y el sorgo var. Cow Vittles. La disminución en los días al segundo corte pudo deberse a que aquí las plantas ya contaban con un sistema radicular desarrollado antes de iniciar su crecimiento, mientras que en el primer corte se partió desde la etapa de semilla, ya que las temperaturas promedio de ambos cortes fueron similares, alrededor de $27^{\circ} \mathrm{C}$. Sin embargo, se sabe que más que otros factores climáticos, es la temperatura la
Cuadro 1. Días al corte y unidades calor requeridas de 13 genotipos forrajeros bajo condiciones de riego en los Valles Centrales de Oaxaca, durante el ciclo primavera-verano de 1998.

\begin{tabular}{|c|c|c|c|c|}
\hline \multirow[t]{2}{*}{ Genotipo* } & \multicolumn{2}{|c|}{ Días a } & \multicolumn{2}{|c|}{ Unidades calor a } \\
\hline & 1er corte & $2^{\text {do }}$ corte & 1er corte & $2^{\text {do }}$ corte \\
\hline Sweet Sioux (S) & 70 & 57 & 853,5 & 688,5 \\
\hline Beefbuilder-T (S) & 63 & 58 & 763,5 & 704,5 \\
\hline Grazer (S) & 63 & 54 & 763,5 & 655,0 \\
\hline Dine-a-Mite (S) & 62 & 47 & 748,5 & 572,0 \\
\hline Domor (S) & 62 & 47 & 748,5 & 572,0 \\
\hline Cow Vittles (S) & 62 & 43 & 748,5 & 524,5 \\
\hline ICMS7704 (M) & 61 & 44 & 733,5 & 539,5 \\
\hline NELC C4 (M) & 61 & 43 & 733,5 & 525,0 \\
\hline ICMS $8283(\mathrm{M})$ & 61 & 43 & 733,5 & 525,0 \\
\hline ICMH83401(M) & 59 & 44 & 704,0 & 540,5 \\
\hline ICMH 423 (M) & 60 & 38 & 719,0 & 465,5 \\
\hline ICTP 8203 (M) & 60 & 38 & 719,0 & 465,5 \\
\hline Pabellón88(M) & 54 & 24 & 640,5 & 304,0 \\
\hline
\end{tabular}

$* \mathrm{~S}=$ sorgo, $\mathrm{M}=$ mijo perla.

que determina la duración de las etapas fenológicas (Duncan 1978).

La relación entre Unidades Calor y días a los cortes fue lineal $(\mathrm{P} \leq 0,01)$, con una correlación muy alta (Figura 1). Se han reportado relaciones lineales entre las unidades calor estimadas por el método residual y la duración del período vegetativo para distintos cultivos C4 (Newman 1971; Ruiz 1988).

\section{Eficiencia de producción de materia seca}

La eficiencia de producción de materia seca fue la más alta en los sorgos Domor y Sweet Sioux y Beefbuilder-T en el primer corte, pero el mijo NELC-C4

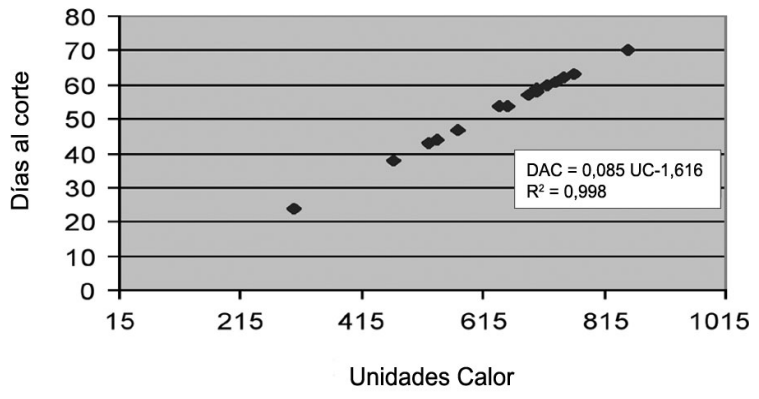

Figura 1. Relación entre días al corte (DAC) y unidades calor (UC) para seis genotipos de sorgo y siete de mijo perla bajo condiciones de riego. 
Cuadro 2. Eficiencia de producción de materia seca por corte y valor promedio para 13 genotipos forrajeros bajo condiciones de riego en los Valles Centrales de Oaxaca, primavera-verano, 1998.

\begin{tabular}{|c|c|c|c|}
\hline \multirow[t]{2}{*}{ Genotipo } & \multicolumn{3}{|c|}{ Eficiencia (kg/ha/día) } \\
\hline & 1er corte & $2^{\text {do }}$ corte & Promedio \\
\hline Domor (S) & $250 \mathrm{a}^{* *}$ & $240 \mathrm{~b}^{* *}$ & $245 \mathrm{a}^{* *}$ \\
\hline Sweet Sioux (S) & $230 \mathrm{ab}$ & $280 \mathrm{ab}$ & $255 \mathrm{a}$ \\
\hline NELC C4 (M) & $220 \mathrm{abc}$ & $170 \quad \mathrm{~cd}$ & $195 \mathrm{~b}$ \\
\hline Beefbuilder-T (S) & $220 \mathrm{abc}$ & $270 \mathrm{ab}$ & $245 \mathrm{a}$ \\
\hline ICTP $8203(\mathrm{M})^{*}$ & 200 bcd & 150 & $175 \mathrm{~b}$ \\
\hline ICMS $8283(\mathrm{M})$ & 200 bcd & 160 & $180 \mathrm{~b}$ \\
\hline ICMH $423(\mathrm{M})$ & 190 bcd & $190 \mathrm{c}$ & $190 \mathrm{~b}$ \\
\hline Grazer (S) & $190 \mathrm{bcd}$ & $250 \mathrm{~b}$ & $220 \mathrm{ab}$ \\
\hline Dine a Mite (S) & 190 bcd & $290 \mathrm{a}$ & $240 \mathrm{a}$ \\
\hline ICMH $83401(\mathrm{M})$ & 180 & 160 & $170 \mathrm{~b}$ \\
\hline ICMS 7704 (M) & 170 & 150 & $160 \mathrm{bc}$ \\
\hline Cow Vittles (S) & 170 & $260 \mathrm{ab}$ & $215 \mathrm{ab}$ \\
\hline Pabellón 88 (M) & 140 & 140 & $140 \quad \mathrm{c}$ \\
\hline
\end{tabular}

$* \mathrm{~S}=$ sorgo, $\mathrm{M}=$ mijo perla

** Medias con letras iguales dentro de la misma columna no son estadísticamente diferentes (Tukey, 0,05).

también quedó incluido en ese grupo (Cuadro 2). Sin embargo, para el segundo corte, solamente Sweet Sioux y Beefbuilder-T permanecen en el grupo de mayor eficiencia, destacando Dine-a-mite.

En promedio, los genotipos de sorgo fueron los más eficientes, especialmente Sweet Sioux y Domor, este último por mostrar poca variación entre cortes. Se hace notar que Domor habría quedado fuera del primer grupo de significancia si solamente se hubiera tomado en cuenta la producción total de materia seca.

La EPMS del segundo corte se correlacionó significativamente $(P \leq 0,05)$ con el IAF, con una $r=0,927$, más no la del primer corte. En la India, dos de cuatro variedades de sorgo evaluadas en tres fechas de siembra produjeron eficiencias máximas de 159-182 kg/ha/día de la siembra a $50 \%$ de floración, lo cual se asoció a mayores IAF (Krishnamurty 1973).

\section{Calidad del forraje}

Como se esperaba los mijos mostraron un mayor porcentaje de hojas (Robles 1983) y tallos (Huda 1987) en ambos cortes, incrementándose los valores de ambos parámetros en todos los genotipos en el segundo corte
(Cuadro 3). Se observó una correlación significativa $(\mathrm{P} \leq 0,01), \mathrm{r}=0,83$, entre $\%$ de hojas y número de tallos. Sin embargo, la correlación entre \% de hojas y la eficiencia de producción tendió a ser negativa, aunque no significativa, especialmente en el segundo corte. Una relación similar se observó con la materia seca total producida.

En general, a mayor porcentaje de tallos, la calidad disminuye (White et al. 1981) pero la alta correlación obtenida en el presente estudio indica que si no hay muchos tallos, tampoco habrá un número elevado de hojas. Los rangos de \% de hojas para mijos variaron de 28,5 42,8 , mientras que para sorgo variaron entre 20,3 y 29,0 $\%$. Se ha reportado que el \% de hojas de seis genotipos del género Pennisetum varió entre 27 y 57 \% (Schank et al. 1993).

La palatabilidad y calidad del forraje son características importantes, ya que la aceptación y valor nutritivo de los piensos se relaciona directamente con la ganancia en peso de los animales, pues se sabe que a mayor porcentaje de hojas y menor edad, mayor valor nutritivo y mayor palatabilidad (Báez 1988). Sin embargo, el uso de agua también puede incrementarse al existir una mayor superficie para transpiración (Winkel et al. 2001).

Cuadro 3. Porcentaje de hojas y número de tallos al primer y segundo corte para 13 genotipos forrajeros bajo condiciones de riego en los Valles Centrales de Oaxaca, durante el ciclo primavera-verano de 1998.

\begin{tabular}{|c|c|c|c|c|}
\hline \multirow[t]{2}{*}{ Genotipo } & \multicolumn{2}{|c|}{ \% de hojas } & \multicolumn{2}{|c|}{ Número de tallos $/ \mathrm{m}^{2}$} \\
\hline & $1^{\mathrm{er}}$ corte & $2^{\text {do }}$ corte & 1er $^{\text {er }}$ corte & $2^{\text {do }}$ corte \\
\hline Pabe & $55, \pi$ & 42 & & \\
\hline ICMH & $33,00 \mathrm{ab}$ & $31,50 \mathrm{bcd}$ & $191 \mathrm{a}$ & $204 \mathrm{ab}$ \\
\hline ICMS & $32,75 \mathrm{ab}$ & $38,25 \mathrm{ab}$ & 151 & $199 \mathrm{ab}$ \\
\hline ICMS & 32,50 & 37,0 & 20 & \\
\hline ICM & 30,75 & $31,75 \mathrm{bcd}$ & 169 & \\
\hline ICTP & $30,75 \mathrm{abc}$ & $28,50 \mathrm{cde}$ & 136 & $186 \mathrm{abc}$ \\
\hline $\mathrm{NE}$ & 30 & & & \\
\hline Graze & 25,00 & $26,75 \mathrm{de}$ & 116 & $149 \mathrm{bc}$ \\
\hline Cow Vitt & 24,75 & $29,00 \mathrm{cde}$ & $69 \mathrm{~d}$ & $99 \mathrm{~d}$ \\
\hline Dine a Mite $(S)$ & $23,25 \mathrm{~cd}$ & 27,25 de & $116 \mathrm{~b}$ & $133 \mathrm{~b}$ \\
\hline Beefbuilder-T (S)* & $20,75 d$ & $23,25 \mathrm{e}$ & $80 \mathrm{~d}$ & $94 \mathrm{~d}$ \\
\hline Sweet Sioux (S) & $20,50 \mathrm{~d}$ & $24,75 \mathrm{de}$ & $86 \mathrm{~cd}$ & $110 \mathrm{~cd}$ \\
\hline Domor (S) & $20,00 \mathrm{~d}$ & $26,75 \mathrm{de}$ & $93 \mathrm{~cd}$ & $132 \mathrm{bcd}$ \\
\hline
\end{tabular}

$* \mathrm{~S}=$ sorgo, $\mathrm{M}=$ mijo perla

** Medias con letras iguales dentro de la misma columna no son estadísticamente diferentes (Tukey, 0,05). 


\section{Indices de productividad}

En el Cuadro 4 se presentan los índices de productividad obtenidos. De acuerdo al IP, en el primer corte sobresalieron los mijos ICMS 8283, ICMH 423 y NELC C4. Sin embargo, no existieron diferencias significativas entre los valores mayores del IPM para sorgos y mijos. En el segundo corte se dio una situación similar, es decir de acuerdo al IP sobresalen los mijos, pero de acuerdo al IPM sobresalen los sorgos, aunque en este caso si se dieron diferencias significativas. Esta condición prevalece si se hace el promedio por los dos cortes. La principal razón es que los mijos mostraron un mayor IC que los sorgos, pero al considerar la eficiencia, los sorgos fueron mas productivos.

Sin embargo, el IP no mostró correlaciones significativas $(\mathrm{P}>0,05)$ con la producción de materia seca por corte (PMS) ni con la EPMS, mientras el IPM mostró correlaciones positivas y altamente significativas $(\mathrm{P} \leq$ 0,01). La correlación entre IPM y PMS por corte mostró valores de 0,938 a 0,944, mientras que la de IPM con EPMS produjo valores de 0,987 a 0,997, lo cual implica que existe una relación mas estrecha del IPM con los atributos deseables en una variedad forrajera: alta eficiencia de producción de materia seca y mayor calidad.
En la Figura 2 se presenta la relación entre el IPM y EPMS y, así como entre IPM y PMS, observándose una relación más cercana a la lineal entre las dos primeras variables. La mayor dispersión observada en el segundo par de variables podría deberse a una mayor variación de los valores de PMS con relación a los de EPMS, ya que al hablar de eficiencia se están transformando éstos al dividirlos por los días al corte, lo cual reduce su rango.

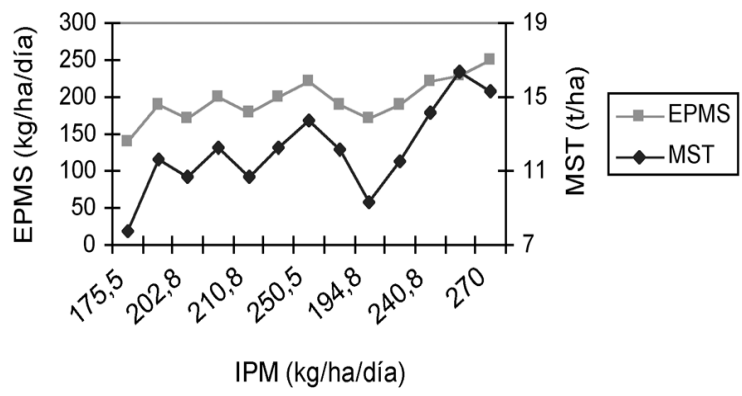

Figura 2. Relación entre el Índice de Productividad Modificado (IPM), la Materia Seca Total (MST) y la Eficiencia de Producción de Materia Seca (EPMS) para el primer corte en variedades forrajeras de sorgo y mijo.

Cuadro 4. Índices de productividad por variedad y por corte para 13 genotipos forrajeros bajo condiciones de riego en los Valles Centrales de Oaxaca, durante el ciclo primavera-verano de 1998.

\begin{tabular}{|c|c|c|c|c|}
\hline \multirow[t]{2}{*}{ Genotipo } & \multicolumn{2}{|c|}{ Índice de Prod. de Stokofv } & \multicolumn{2}{|c|}{ Índice de Prod. Modificado } \\
\hline & 1er $^{\mathrm{er}}$ corte & $2^{\text {do }}$ corte & $1^{\mathrm{er}}$ corte & $2^{\text {do }}$ corte \\
\hline Pabellón 88 (M) & $43,26 \mathrm{a}^{* *}$ & $46,18 \mathrm{a}^{* *}$ & $175,5 \mathrm{c}^{* *}$ & $182,8 \mathrm{~d}^{* *}$ \\
\hline ICMH 423 (M) & 44,64 a & $38,92 \mathrm{~d}$ & $223,0 \mathrm{ab}$ & $221,5 \mathrm{c}$ \\
\hline ICMS 7704 (M) & 43,49 a & 44,93 a & $202,8 \mathrm{~b}$ & $188,3 \mathrm{~d}$ \\
\hline ICMS $8283(\mathrm{M})$ & 44,81 a & 44,04 a & $232,5 \mathrm{ab}$ & $197,0 \mathrm{~cd}$ \\
\hline ICMH 83401 (M) & $41,48 \mathrm{a}$ & $39,06 \mathrm{~d}$ & $210,8 \mathrm{~b}$ & $191,8 \mathrm{~cd}$ \\
\hline ICTP 8203 (M) & 43,06 a & 34,09 e & $230,8 \mathrm{ab}$ & $178,5 \mathrm{~d}$ \\
\hline NELC C4 (M) & 44,26 a & $42,97 \mathrm{abc}$ & $250,5 \mathrm{a}$ & $205,8 \mathrm{~cd}$ \\
\hline Grazer (S) & $37,17 \mathrm{~b}$ & $40,12 \mathrm{dc}$ & $215,0 \mathrm{ab}$ & 276,8 a \\
\hline Cow Vittles (S) & $34,14 \mathrm{~b}$ & $40,28 \mathrm{dc}$ & $194,8 \mathrm{bc}$ & $289,0 \mathrm{a}$ \\
\hline Dine a Mite (S) & $34,86 \mathrm{~b}$ & $41,06 \mathrm{bcd}$ & $213,3 \mathrm{~b}$ & 317,8 a \\
\hline Beefbuilder-T (S)* & * $34,99 \mathrm{~b}$ & $39,06 \mathrm{~d}$ & 240,8 a & 293,3 a \\
\hline Sweet Sioux (S) & $36,91 \mathrm{~b}$ & $40,52 \mathrm{bcd}$ & $250,5 \mathrm{a}$ & $304,8 \mathrm{a}$ \\
\hline Domor (S) & $35,27 \mathrm{~b}$ & $38,23 \mathrm{~d}$ & $270,0 \mathrm{a}$ & $266,8 \mathrm{ab}$ \\
\hline
\end{tabular}

* $\mathrm{S}=$ sorgo, $\mathrm{M}=$ mijo perla

** Medias con letras iguales dentro de la misma columna no son estadísticamente diferentes (Tukey, 0,05). 
Los valores de IP promedio para los dos cortes variaron desde 36,8 hasta 44,7, los cuales pueden considerarse bajos, pues se han reportado valores de IP de 54,3 a 56,2 para variedades de trigo (Triticum aestivum) de diferente altura y para maíz sembrado a distintas densidades IP's de 63,5 a 72,8 para el rango de poblaciones de 12.500-100.000 plantas/ha (Stoskopfv 1981). Por lo que respecta al IPM, mostró valores de 179,2 a 277,7, un rango mucho más amplio que se asoció mejor con los rangos promedio de EPMS observados, 140-245 kg/ha/día.

\section{CONCLUSIONES}

El número de días al corte es una característica de importancia primordial cuando se trata de identificar variedades forrajeras. Los mijos resultaron más precoces que el grupo de genotipos de sorgo en cuanto a los días al primer corte. Para el segundo corte o soca, los días a $50 \%$ de floración disminuyeron, especialmente en los mijos y en el sorgo var. Cow Vittles.

La utilización de índices de eficiencia de la producción de materia seca es una manera de optimizar los recursos disponibles, particularmente el agua de riego. En promedio de dos cortes, los genotipos de sorgo fueron los más eficientes para producir materia seca, especialmente Sweet Sioux y Domor, éste último con menor variación de eficiencia entre cortes. Los mijos más eficientes, NELC C4 y ICMH 423, redujeron su rendimiento en un $46 \%$ en el segundo corte, pero podrían ser utilizados por campesinos que tengan urgencia de forraje, y agua o tiempo para sólo un corte.

Se observó una correlación significativa $(\mathrm{P} \leq 0,01)$ entre \% de hojas y número de tallos, encontrándose valores superiores de estos parámetros en el grupo de los mijos.

Dado que la densidad de siembra puede determinar el grado de ahijamiento, para incrementar la calidad del forraje, se debe determinar la densidad óptima de siembra en los materiales más sobresalientes.

La utilización de índices relacionados con atributos deseables en los materiales genéticos constituye una alternativa para la identificación expedita de las variedades con mayor potencial.

El IPM superó al IP en su capacidad de discriminar los materiales con mayor eficiencia de producción de materia seca y calidad, por lo que se recomienda su utilización en la selección de variedades forrajeras bajo condiciones de riego restringido.

\section{LITERATURA CITADA}

BÁEZ, G. A. D. 1988. Efecto de la etapa de corte sobre la producción y calidad del forraje de mijo perla. Informe anual del CIFAP-Aguascalientes. INIFAP-SARH, México. $130 \mathrm{p}$.

BANKS, S.; STEWART, T. 1998 Forage pearl millet. Factsheet 126. Ministry of agriculture and Food, Ontario, Canadá. Queens printer. sp.

BEETS, W. C. 1985. Rational crop selection in the tropics. Span 28(1): 4-6.

BOHN, P. J.; BROWN, R. H.; ARKIN D E. 1988. In vitro dry matter digestibility, leaf anatomy and fibber concentration of a hybrid between $\mathrm{C} 3$ y C3-C4 Panicum species. Crop Sci (28):332-336.

BITTMA, S. G.; SIMPSON, M.; MIR, Z. 1988. Leaf senescence and seasonal decline in nutritional quality of three temperate forage grasses as influenced by drought. Crop Sci. 28: 546-552.

CASTRO, N. J.; ORTÍZ, J.; MENDOZA, MA DEL C.; ZAVALA, C. F. 2000. Producción de biomasa en líneas de sorgo como respuesta al estrés hídrico. Rev. Fitotec. Mex. 23: 321-334.

CHAPMAN, D. F.; LEMAIRE, G. 1993. Morphogenetic and structural determinants of plant regrowth after defoliation. Proc XVII Int. Grass Cong., Palmerston North, New Zealand: 95-104.

CHERNEY, J. H.; MARTIN, G. X. 1980. Biological, chemical morphological and anatomic interelationships of forage quality in small grain cereals. Amer. Soc. Agron. Madison, USA. p. 370-371.

CARRILLO, J. C.; RUIZ, J. 2004. Producción de forraje en sorgo y mijo: Variables de crecimiento. Agronomía Mesoamericana 15: 69-76.

CIAN. 1984. El mijo perla: Un nuevo forraje para La Comarca Lagunera. Folleto Técnico No. 8, Campo Agrícola Experimental Laguna, Matamoros Coahuila, México. $35 \mathrm{p}$.

DUNCAN, W G. Sugar cane. 1978. In: L. T. Evans ed. Crop Physiology: Some case histories. Cambridge University Press, London, England. p. 23-50.

FALES, S. 1986. Effects of temperature on fiber concentration, composition and in vitro digestion kinetics of tall fescue.Agron J. 78:963-966.

FISCHER, R A. 1980. Influence of water stress on crop yield in semiarid region. In: N. C. Turner; P. J Kramer eds. Adaptation of plants to water and high temperature stress. Wiley, New York. p. 323-339. 
GLIESSMAN, S. R. 2000. Agroecology: Ecological processes in sustainable agriculture. Lewis Publishers, Boca raton, Florida, USA. 357 p.

GONZÁLEZ, A.; MARTÍN, I.; AYERBE, L. 1999. Barley yield in water-stress conditions: The influence of precocity, osmotic adjustment and stomatal conductance. Field Crops Res. 62:23-34.

HIRASAWA T.; HSIAO, T. 1999. Some characteristics of reduced leaf photosyntesis at midday in maize growing in the field. Field Crops Res. 62:53-62.

HOPKINS, W. G. 1999. Introduction to plant physiology. $2^{\mathrm{a}}$. Ed. Willey Inc., New York. 272 p.

HUDA, A K S. 1987. Simulating yields of sorghum and pearl millet in the semi-arid tropic. Field Crops Res. 15:309325 .

KRISHNAMURTY, K. 1973. Investigations on the structure of yield in cereals (maize y sorghum). Agronomy Department, University of Agricultural Sciences, Bangalore, India. 374 p.

LÓPEZ, J.; ROJO., J.; CANO, M.; ARREDONDO, C.; RUIZ, J. 1992. Los sistemas de producción agropecuaria en los Valles Centrales de Oaxaca II. Identificación y funcionamiento. Resúmenes del Coloquio Mesoamericano sobre Sistemas de Producción y Desarrollo Agrícola. 22-26 de junio, Texcoco, Mex. CP-CEDERU, ORSTOM. p. 68.

LOWENBERG-DEBOER, J.; KRAUSE. M.; DEUSON, R.;REDDY, K C. 1991. Simulation of yield distributions in millet-cowpea intercropping. Agric. Sys. 36:471-487.

MCCOLLUM, T.; BEAN, B. 2003. Comparative value of silages based on digestibility. Asweb-105, Texas Coop. Extension, Texas A\&M University. USA. 57 p.

MUIR, J. P.; PROSTKO, E.; STOKES, S. 1999. The effect of dairy manure on summer annual grasses grown as alternative silages on the cross timbers. Stephenville Agric. Res. and Ext. Center. University of Texas A\&M. 8 p.

NEWMAN, J. E. 1971. Measuring corn maturity with heat units. Crops and Soils Mag. 23:11-14.

NÚÑEZ, G.; CANTÚ-BRITO, E. 2000. Forage yield, chemical composition and digestibility of brown midrib sorghum x sudan forage in northern Mexico. Tec. Pecu. Mex. 38(3): 177-187

PINKERTON, B. W.; UNDERSANDER, D. J.; PARDUE; F. E.; WEBSTER, H. W.; OLSON, L. W.; HUDSON, L. W. 1991. Interpretation of feed and forage laboratory reports. Forage Leaflet 11: 1-6. Cooperative Extension Service, Clemson University, South Carolina, USA.
RAPER C. D.; KRAMER, P. J. 1983. Crop reactions to water and temperature stresses in humid, temperate climates. Western Press, Boulder, USA. 384 p.

ROBLES, R. 1983. Producción de granos y forrajes. Ed. Limusa, México, D. F., cuarta edición. 460 p.

RUIZ, J. 1988. Evaluación de tres variedades de mijo perla a tres densidades de población en los Valles Centrales de Oaxaca. Informe Anual de Investigación, INIFAP-CAEVOAX, Sto. Domingo Barrio Bajo, Etla. México. p. 2532

RUIZ, J. 1997. Fisiotecnia vegetal. Apuntes para la maestría en productividad de agrosistemas. Instituto Tecnológico Agropecuario No. 23, Nazareno Xoxocotlán Oaxaca, México. 137 p.

SCHANK, S. C.; CHYNOWETH, D. P.; TURICK, C. E.; MENDOZA, E. 1993. Napiergrass genotypes and plantparts for biomass energy. Biomass \& Energy 4:1-7.

SECRETARÍA DE AGRICULTURA, GANADERÍA Y DESARROLLO RURAL (SAGDR). 1995. Anuario estadístico de la producción agrícola de los Estados Unidos Mexicanos. Centro de estadística agropecuaria. SAGAR, México, D. F: 178 p.

STATISTICAL ANALYSIS SYSTEM, 1994. SAS Institute, North Carolina, USA. 494 p.

STICKLER, F.C.; WEARDEN, S.; PAULI A W. 1961. Leaf area determination in grain sorghum. Agron. J. 53: 187188.

STOSKOPFV, N. C. 1981. Understanding crop production. Reston Publishing Co. Inc., Reston, Virginia. 433 p.

TAPIA V., M. 1985. Cultivos de alternativa: una posible solución al problema de la sequía en la Mixteca Oaxaqueña [Tesis de licenciatura]. Morelia, México: Universidad Michoacana de San Nicolás Hidalgo. 150 p.

WHITE, L. M.; HARTMAN, G. P.; BERGMAN, J. W. 1981. In vitro digestibility crude protein and phosphorus content of winter wheat, spring wheat and oat cultivar in Eastern Montana. Agron J. 73:117-121.

WILSON, J. R.; BROWN, R. H.; WINDHAM, W. R. 1983. Influence of leaf anatomy on the dry matter digestibility of $\mathrm{C} 3, \mathrm{C} 4$ and $\mathrm{C} 3 / \mathrm{C} 4$ Panicum species. Crop Sci. 23:141-146.

WINKEL T.; PAYNE, W.;RENNO, J. F. 2001. Ontogeny modifies the effects of water stress on stomatal control, leaf area duration and biomass partitioning of Pennisetum glaucum. New Phytol. 149(1):71-82. 